\title{
Evaluating our ability to predict the structural disruption of RNA by SNPs
}

\author{
Justin Ritz ${ }^{\dagger}$, Joshua S Martin ${ }^{\dagger}$, Alain Laederach ${ }^{*}$ \\ From SNP-SIG 2011: Identification and annotation of SNPS in the context of structure, function and disease \\ Vienna, Austria. 15 July 2011
}

\begin{abstract}
The structure of RiboNucleic Acid (RNA) has the potential to be altered by a Single Nucleotide Polymorphism (SNP). Disease-associated SNPs mapping to non-coding regions of the genome that are transcribed into RiboNucleic Acid (RNA) can potentially affect cellular regulation (and cause disease) by altering the structure of the transcript. We performed a large-scale meta-analysis of Selective 2'-Hydroxyl Acylation analyzed by Primer Extension (SHAPE) data, which probes the structure of RNA. We found that several single point mutations exist that significantly disrupt RNA secondary structure in the five transcripts we analyzed. Thus, every RNA that is transcribed has the potential to be a "RiboSNitch;" where a SNP causes a large conformational change that alters regulatory function. Predicting the SNPs that will have the largest effect on RNA structure remains a contemporary computational challenge. We therefore benchmarked the most popular RNA structure prediction algorithms for their ability to identify mutations that maximally affect structure. We also evaluated metrics for rank ordering the extent of the structural change. Although no single algorithm/metric combination dramatically outperformed the others, small differences in AUC (Area Under the Curve) values reveal that certain approaches do provide better agreement with experiment. The experimental data we analyzed nonetheless show that multiple single point mutations exist in all RNA transcripts that significantly disrupt structure in agreement with the predictions.
\end{abstract}

\section{Background}

RNA (Ribonucleic Acid) is a ubiquitous messenger of genetic information in the cell and plays a central role in the regulation of molecular processes [1-5]. Unlike DNA, RNA is generally single stranded and has a high propensity to fold into functionally important structures [6-10]. These structures can be significantly disrupted by mutations including Single Nucleotide Polymorphisms (SNPs) $[11,12]$. Genome-Wide Association Studies (GWAS) regularly identify disease-associated SNPs in non-coding regions of the genome. Disease-associated SNPs do not necessarily directly reveal the molecular cause of the disease and require further analysis [11,13-15].

A majority of the genome is transcribed into RNA $[16,17]$; as a result a majority of genetic mutations will also be transferred to the transcriptome. From a structural

\footnotetext{
* Correspondence: alain@unc.edu

† Contributed equally

Department of Biology, University of North Carolina, Chapel Hill, NC, 27599,
} USA

\section{Biomed Central}

(c) 2012 Ritz et al; licensee BioMed Central Ltd. This is an Open Access article distributed under the terms of the Creative Commons Attribution License (http://creativecommons.org/licenses/by/2.0), which permits unrestricted use, distribution, and reproduction in any medium, provided the original work is properly cited. highly structured RNAs (e.g. the Ribosome, tRNAs, self splicing introns, RNAse P) and RNAs that potentially adopt multiple conformations (e.g. mRNAs and non-coding RNAs) $[3,4,18]$. Structured RNAs are under significant evolutionary pressure to adopt a single, functional conformation [19]. However, mRNAs and non-coding RNAs are not necessarily evolved to adopt a single conformation but rather adopt an ensemble of conformations [20-23]. We have recently found specific disease-associated mutations that alter the ensemble partitioning of mRNA affecting gene regulation and thus cause disease [24]. Thus, structure is likely an important functional feature even in RNAs traditionally thought of as "unstructured."

Algorithms to evaluate the structural and functional consequences of mutations on proteins (e.g. PolyPhen and SIFT) are commonly used to assess the potential deleterious effects of mutations [25-27]. In addition, several groups are actively developing web servers to compute the potential deleterious effects of SNPs on RNA structure 
and function $[28,29]$. The structural basis for deleterious mutations to a structured protein is rationalized through an understanding of protein folding. For example, replacing a hydrophobic residue in the hydrophobic core of a protein with a hydrophobic amino acid will likely cause the protein to misfold [26,27]. In RNA however, the physico-chemical properties of the four-nucleotides are not as diverse as the amino acids. Furthermore, RNA does not fold through the formation of a hydrophobic core [4]. Instead the structure is a complex network of base-pairing and stacking interactions $[3,8]$. To observe a large conformational change in an RNA, the mutation must not only disrupt an existing base-pair, but also favor a completely alternative base-pairing network. The functional consequences of structure disruption depend on whether the affected region is involved in important regulatory interactions. In certain cases, small local changes in the RNA structure may have functional consequences $[15,30]$. In this manuscript we are interested in identifying the mutations that globally affect RNA structure and are thus likely to have significant functional consequences.

We initially interrogate high-throughput SHAPE chemical mapping of multiple non-coding RNAs and associated single point mutations $[31,32]$. We aim to determine whether single point mutations, like in proteins, can significantly alter the structure of the RNA. We then evaluate the performance of multiple RNA structure prediction algorithms to determine the optimal strategy for identifying the mutations that disrupt RNA structure. As GWAS (Genome Wide Association Studies) continue to focus more on non-coding regions of the genome, it will become increasingly important to have accurate algorithms for assessing the potential deleterious consequences of SNPs on the transcriptome.

\section{Results and discussion}

\section{Single mutations disrupt RNA structure}

To better understand the potential effects of SNPs on a large RNA we consider the Boltzmann sampled suboptimal ensemble of the Vibrio vulnificus Adenine Riboswitch (Figure 1A) [33,34]. Projecting these structures onto the first two principal components of their structural space as described previously [24], reveals four major clusters (Figure 1A). The Adenine Riboswitch is so named as the aptamer domain (highlighted in light magenta in Figure 1A) binds Adenine. It is one of the few Riboswitches that activates gene expression upon ligand binding [35-37].

The "on" and "off" conformations of the Riboswitch are present in the Boltzmann ensemble of the WT sequence (Figure 1A, green and magenta clusters, respectively). This is consistent with recent models that suggest that Adenine riboswitching is kinetically controlled at the transcriptional level [35]. Moreover, two other conformations (cyan and red clusters, Figure 1A) are not highly populated in the WT ensemble. If we repeat the Boltzmann sampling procedure for a sequence containing the $\mathrm{C} 77 \mathrm{G}$ mutation (Figure 1B), we see a drastic shift in the ensemble favoring the cyan and red conformations. A majority of mutations, however, are like the U39A mutation and have very little effect on the suboptimal ensemble (Figure 1C).

To experimentally validate the prediction made by suboptimal sampling made in Figures $1 \mathrm{~A}-\mathrm{C}$, we queried the SNRNASM (Single Nucleotide Resolution Nucleic Acid Structure Mapping) archive as well as the RNA Mapping Database (RMDB, http://rmdb.stanford.edu) for chemical mapping data of the Adenine Riboswitch [38]. We found SHAPE chemical mapping data for the WT, C77G and U39A transcripts under identical solution conditions (10 $\mathrm{mM} \mathrm{MgCl} 2$ and $100 \mathrm{mM} \mathrm{KCl}$ ). This data provides single nucleotide resolution measurements of base-pairing in the Riboswitch [39]. A high normalized SHAPE reactivity indicates high flexibility and thus low probability of basepairing, while low reactivity indicates high likelihood of base-pairing $[40,41]$. The data in Figure 1D therefore experimentally validates the predictions made in Figures 1A-C. We see that the C77G (red trace) is significantly different from the black (WT) and blue (U39A) traces, consistent with a large shift in the predominant structures in the ensemble. The significant increase in SHAPE reactivity in residues 32-43 and 62-68 are consistent with the hairpin structure represented by cyan cluster.

We compute the experimental Structure Disruption Coefficient (eSDC) to evaluate the effect of a SNP on the RNA structure as described in the Methods (Equation 1). This value measures the disruptive effect of a SNP on an RNA, the higher it is the greater the structural disruption. In this case it is 2.0 for C77G and 0.1 for U39A. Furthermore, we can use the multiple repeats of the experiments to evaluate the statistical significance (p-value) of these eSDC values, i.e. the probability that we would obtain the value due to noise in the data. For the $\mathrm{C} 77 \mathrm{G}$, the difference is statistically significant ( $p$-value $<0.001$ ) while for U39A it is not ( $p$-value $>0.5$ ).

\section{Systematic eSDC analysis of five non-coding RNAs}

The SNRNASM and RMDB databases contain 470 SHAPE data sets of RNA sequences with single and/or double point mutations relative to WT RNA for five non-coding RNAs under similar monovalent and divalent salt concentrations. We therefore computed eSDC values for these 470 mutations and summarize the results in Figure 2A. In all cases we computed eSDC values relative to the WT sequence to identify single or double mutations that significantly disrupt RNA structure.

The results of our analyses are plotted on Figure 2A and reveal that in all cases certain mutations (e.g. U22G.A196G in FTL, U113A in the Glycine Riboswitch) significantly 

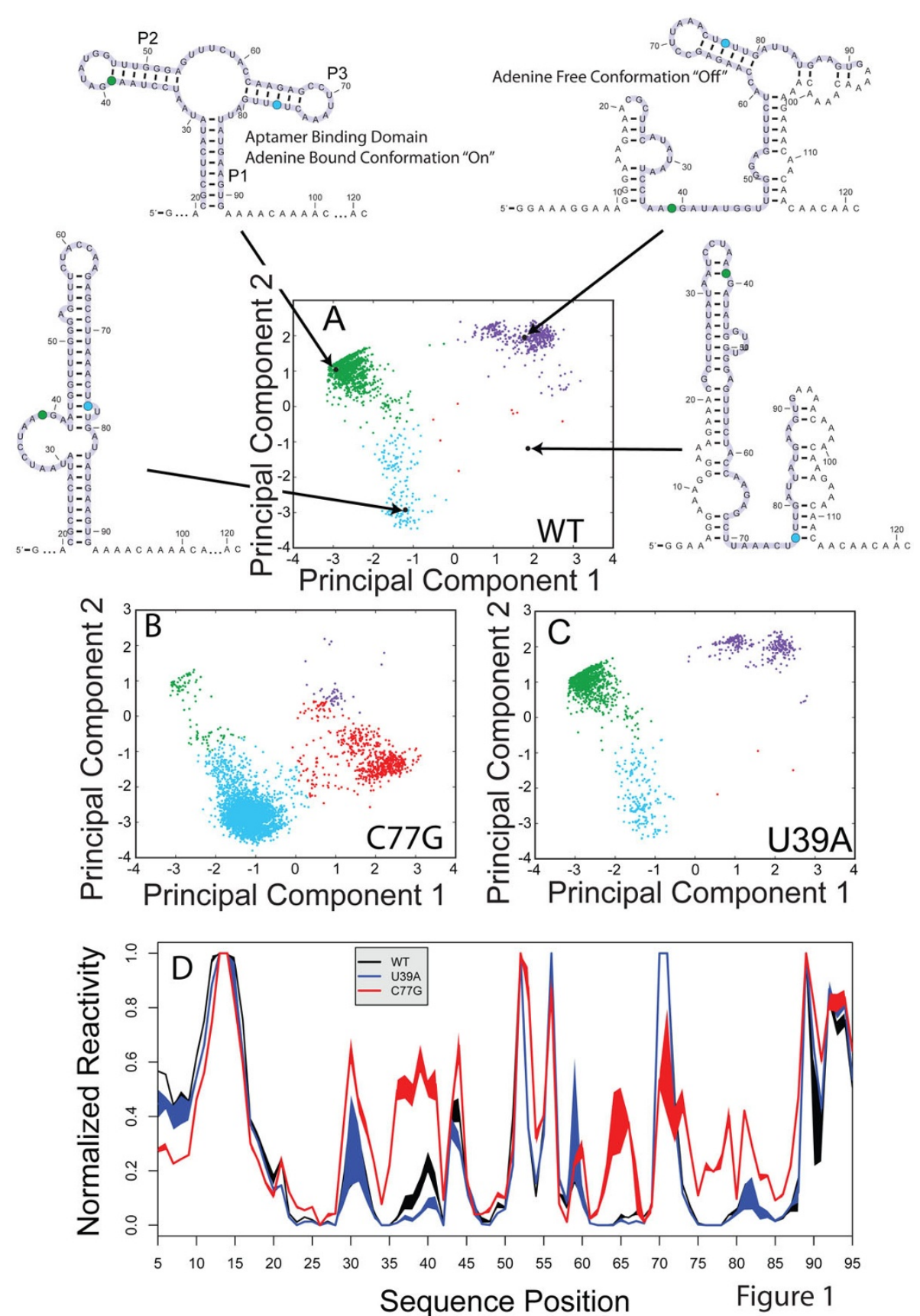

Figure 1 Structural analysis of the Adenine Riboswitch, which is a bacterial regulatory RNA that binds Adenine and controls gene expression $[35,37]$. The RNA adopts two major conformations, the "On" state (Adenine bound) forms three stem loops (P1, P2 and P3), while in the "off state" the site of translation initiation ( $3^{\prime}$ end of the UTR, near the start codon) is structured effectively disrupting translation initiation. A.) Boltzmann suboptimal sampling of the ensemble of possible RNA conformations (as predicted by sFold) projected onto the first two principal components of structure space as determined by a Manhattan distance metric evaluation of the ensemble. Each dot in the diagram is one alternative structure. Representative structures adorn the diagram, and the aptamer domain of the Riboswitch is highlighted in light magenta. The Riboswitch is predicted to adopt four structures, characterized by green, purple, cyan and red dots. The "on" and "off" states of the Riboswitch to the green and magenta cluters, respectively. B.) Boltzmann sampling of the structural ensemble for the C77G containing sequence which indicates a significant shift in partitioning towards the cyan and red conformations. C.) Boltzmann sampling for the U39A mutation which is predicted to have no effect on the partitioning compared to WT. D.) Experimental validation using SHAPE chemistry of the predictions made in A-C, showing that the C77G mutation disrupts the structure of the RNA in a manner consistent with an increase in the population of the cyan cluster. 

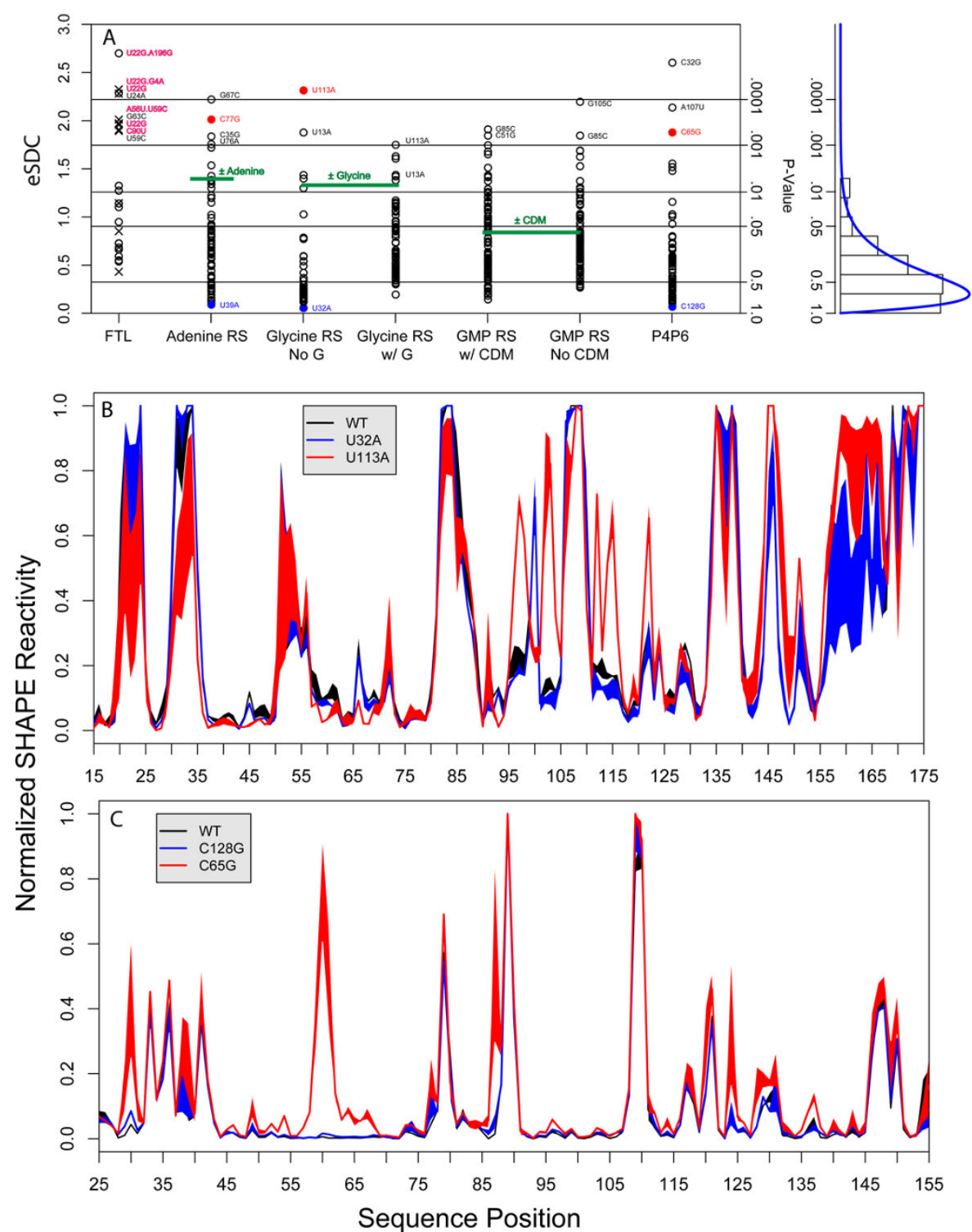

Figure 2

Figure 2 Comprehensive analysis of mutation induced structure disruption in five non-coding RNAs. A.) eSDC (experimental Structure Disruption Coefficient) for 470 single or double mutatants relative to the RNA's WT sequence. eSDC is computed as one minus the Pearson correlation coefficient of the SHAPE profile (mutant to WT) multiplied by the square root of the length of the RNA. We see that most mutations have small eSDC values indicating that they do not significantly disrupt structure. The five RNAs studied are the human FTL 5' UTR (FTL), the V. vulnificus Adenine Riboswitch (Adenine RS), the V. cholera Glycine Riboswitch (Glycine RS with and without Glycine (G) bound), the cyclic di-GMP Riboswitch (bis-(3'-5')-cyclic dimeric guanosine monophosphate Riboswitch with and without cyclic-diguanosine-monophosphate (CDM)) and the P4P6 domain of the L-21 Tetrahymena thermopila group I intron [5,34,35,63]. All data were collected under near physiological solution conditions, i.e. $10 \mathrm{mM} \mathrm{MgCl}$ and $100 \mathrm{mM}$ monovalent. For FTL, hyperferritinemia associated mutations are indicated in magenta. The eSDC values for \pm ligand for the three Riboswitches are indicated with a green horizontal line and represent a "biological" threshold above which a structure change is likely to have a functional consequence. This histogram to the right represents a pairwise "within" eSDC calculation for 6-fold repeats of the SHAPE experiments on the FTL UTR RNA to evaluate the reproducibility and significance ( $p$-value) of eSDC values. B.) SHAPE profiles for the WT, U32A and U113A (black, blue, and red respectively) Glycine Riboswitch in the presence of Glycine showing that the U113A mutation very significantly disrupts structure. C.) SHAPE profiles for WT, C128G and C65G (black, blue, and red respectively) P4P6 group I intron transcripts showing that the C65G globally affects the structure of the RNA. 
disrupt RNA structure. However, a majority of mutations (e.g. U39A and U32A in the Adenine and Glycine Riboswitches) have very small effects on structure. We plotted representative SHAPE data for structurally disruptive (red) and non-disrupting mutations for the Glycine Riboswitch and P4P6 intron in Figures 2B and C, respectively. To evaluate the significance of the structural disruption, we computed the "within" distribution for multiple repeats (6-fold) of the FTL UTR RNA SHAPE data and plot the resulting histogram to the right of Figure 2A. This allows us to determine the expected eSDC values due to the noise in the experimental data, and evaluate the p-value for any given eSDC. Clearly, single point mutations exist that significantly disrupt RNA structure, however a majority of mutations result in no measurable effect.

The FTL UTR data set is particularly interesting, as this RNA is a "RiboSNitch," i.e. an RNA in which specific SNPs can alter structure and cause disease $[24,42]$. In this case, FTL is associated with Hyperferritinemia Cataract Syndrome, a rare genetic disorder that is characterized by early onset cataracts due to excess ferritin in the retina $[43,44]$. We indicate the disease-associated SNPs as magenta text in Figure 2A. All the disease-associated SNPs alter the structure of the RNA significantly ( $\mathrm{p}$-value $<0.001)$.

Three of the RNAs tested in Figure 2A are Riboswitches and undergo a conformational change if ligand is present. We can therefore compute an eSDC value for SHAPE traces in the presence and absence of ligand. We indicate these eSDC values with a green horizontal line in Figure $2 \mathrm{~A}$. The reason this result is important is that the structural change caused by ligand binding to a Riboswitch is sufficient to regulate gene expression $[37,45,46]$. Thus the Riboswitch ligand eSDC value (green line Figure 2A) represents a "biological" threshold above which the structure change is likely to affect function. A particularly important result of this analysis is the identification of multiple SNPs with much larger eSDC values compared to ligand binding in the Riboswitches. Thus, it is likely that a majority of these SNPs will have important functional consequences.

\section{Performance of RNA structure prediction algorithms for RiboSNitch detection}

We chose to benchmark the four software packages illustrated in Figure 3 [23,47-49], as they each have various options to evaluate the ensemble of suboptimal structures. The precise UNIX commands we used to generate the predictions are also indicated in Figure 3. It should also be noted that all of these programs are designed to predict the best secondary structure, and with the exception of RNAmutants are not necessarily optimized for identifying the mutation that most disrupts RNA structure.
We aim to use RNA structure prediction programs to predict the eSDC values determined from the SHAPE data (Figure 4A). Figure 4 illustrates the four metrics applied to the ensemble of structures from each algorithm and used to generate $\mathrm{pSDC}$ values (predicted Structure Disruption Coefficients, Equation 4, methods). This metric is analogous to the eSDC as it allows us to rank order SNPs according to their predicted disruption of RNA structure. All structure prediction programs we tested can compute a Minimum Free Energy (MFE) structure. We represent this as a vector of ones and zeroes, and compute the correlation coefficient between the WT and mutant structures (Figure 4B). Many structure prediction algorithms can also compute the probability of base-pairing (which is more analogous to SHAPE reactivity) by summing the rows or columns of the predicted partition function matrix (Figure 4C) [48,50]. We computed the Z Centroid (Figure 4D) of the partition function as well [51]. Finally, for the algorithms that sample suboptimal structures, we can cluster the resulting ensemble and determine the centroid structure for the most populated cluster (Figure 4E) [23,51].

We found that in general pSDC values are larger than eSDC values. We are most interested in the different algorithms' (Figure 3) and metrics' (Figure 4) ability to rank and identify the mutations that maximally disrupt structure. To evaluate each algorithm's performance we generated Receiver Operator Characteristic (ROC) curves based on the ranking of the 470 mutant RNA's eSDC values (Figure 2A) compared with those ranked by pSDC. Figure 5A plots three representative ROC curves and illustrates that algorithm/SDC metric combinations vary in their predictive performance. The AUC (Area Under the Curve) values reported in Figure $5 \mathrm{~B}$ suggest that the highest performing algorithm is RNAsubopt using a $\mathrm{Z}$ centroid metric (AUC 0.64). The "partition function" for RNAsubopt was obtained by computing the pair probabilities for the first 10,000 suboptimal structures. The AUC values reported in Figure 5B reveal that most algorithm/metric combinations perform similarly and are within the standard error of 0.03 when the experimental data is bootstrapped. eSDC values, and SHAPE data for all mutants analyzed are provided as tables in the additional files. Additional Files 1-8 correspond to the FTL 199, FTL 226, Adenine RS, Glycine RS NoGlyc, Glycine RS wGlyc, GMP RS wCDM, GMP RS NoCDM, and P4P6, respectively.

\section{Conclusions}

RNA is a ubiquitous regulatory molecule in the cell and there is growing evidence that structure is a central component of its function [52,53]. The Riboswitches studied in this manuscript are one of many examples where RNA structure change regulates bacterial metabolism $[46,54,55]$. 


RNAfold -noPS < seq.fa
$>$ outfile.txt

Figure 3 Schematic representation of the four software packages we benchmarked for their ability to predict which mutations in an RNA affect structure most significantly. We chose these packages as they all perform some form of sub-optimal sampling, illustrated with "cartoon" energy landscapes. We also include the precise UNIX commands used to make the predictions.

In the case of the 5' UTR, disease-associated SNPs disrupt structure and deregulate Ferritin levels in the eye, resulting in early onset cataracts [24]. The $T$. thermophila group I intron (P4P6) must fold into its correct three-dimensional structure to self-catalyze its splicing reaction $[8,56]$. In these examples, structure change is central to the RNA's function in the cell.

The data we present in Figures 1 and 2 reveals the extent to which a single point mutation can disrupt RNA structure. Our systematic analysis of 470 mutations on five RNAs reveals that large scale SNP induced structure change is common in RNA and can potentially contribute to disease [24]. Interestingly, all RNA secondary structure prediction algorithms predict that a small subset of mutations will have a large effect on secondary structure. The data we present in Figures 1 and 2 cover a relatively comprehensive set of mutations in each RNA, but are nonetheless limited to five functional molecules. As such, the generalizability of these results will require the analysis of larger experimental data sets as they become available [38].

The mechanism for this change is best illustrated in Figure 1, where we see how a single mutation (in this case $\mathrm{C} 77 \mathrm{G}$ ) can completely alter the thermodynamic folding landscape of the RNA, favoring an alternative conformation. The data we present in Figure 2 suggest that the thermodynamic models used to predict RNA structure are sound, as we find mutations experimentally in all RNAs studied that disrupt structure. All RNA structure prediction algorithms predict that certain 


\section{METRIC}

\section{WT vs. Mutant}
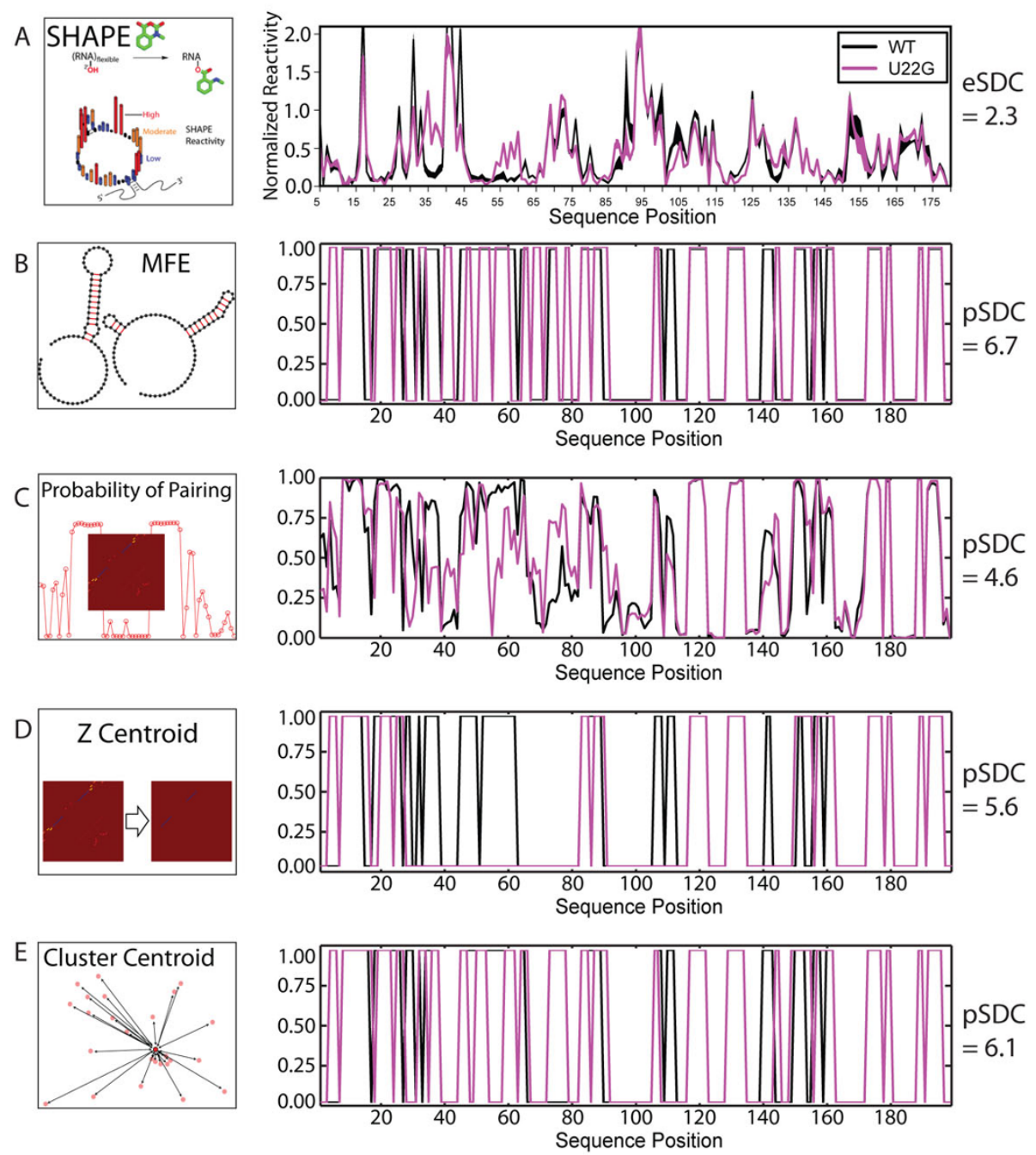

Figure 4 Schematic representation of metrics used to compute pSDC (predicted Structural Disruption Coefficients) based on RNA structure predictions for WT (black) and mutant (magenta). The data here are for the WT, and hyperferritinemia cataract syndrome associated U22G mutant of the FTL 5' UTR. A.) SHAPE experimental data for the WT and U22G mutant UTRs revealing a significant effect of the U22G mutation on the structure of the RNA. An eSDC value of 2.3 is computed for this data. B.) sFold Minimum Free Energy (MFE) probability of base-pairing for the WT (black) and U22G (magenta) containing sequence, one corresponds to not-base-paired and zero paired. We see that the program correctly predicts changes in the 40-60 range as measured by SHAPE. C.) Probability of base-pairing computed as the sum of the rows or columns of the partition function [64]. In this case the partition function is computed using sFold Boltzmann suboptimal sampling and computing the observed frequency of base-pairing [51]. D.) Z Centroid simplification of the partition function and probability of pairing computed by summing the rows or column [51]. E.) Probability of pairing assessed as the cluster centroid structure of the most populated cluster of suboptimal structures, in this case using sFold and k-means clustering as previously described [51].

mutations will significantly disrupt structure. In addition, a recent study of common SNPs in the human genome revealed that these affect local RNA structure [57].

An important result in our analysis of the Riboswitch SHAPE data is the comparison of the eSDC values for mutations relative to ligand induced conformational change (see green lines, Figure 2A). For all three Riboswitches, multiple mutations exist that result in far larger structural changes (as measured by our SDC metric) than ligand binding. This is highly relevant, as ligand binding induced structure change can completely turn on (or off) gene expression translationally and/or transcriptionally [45]. Thus the mutations above the green lines in Figure 2A have even greater potential to regulate cellular function. This means any functional RNA has the potential to be a "RiboSNitch," as there exists mutations that can significantly disrupt its structure. 


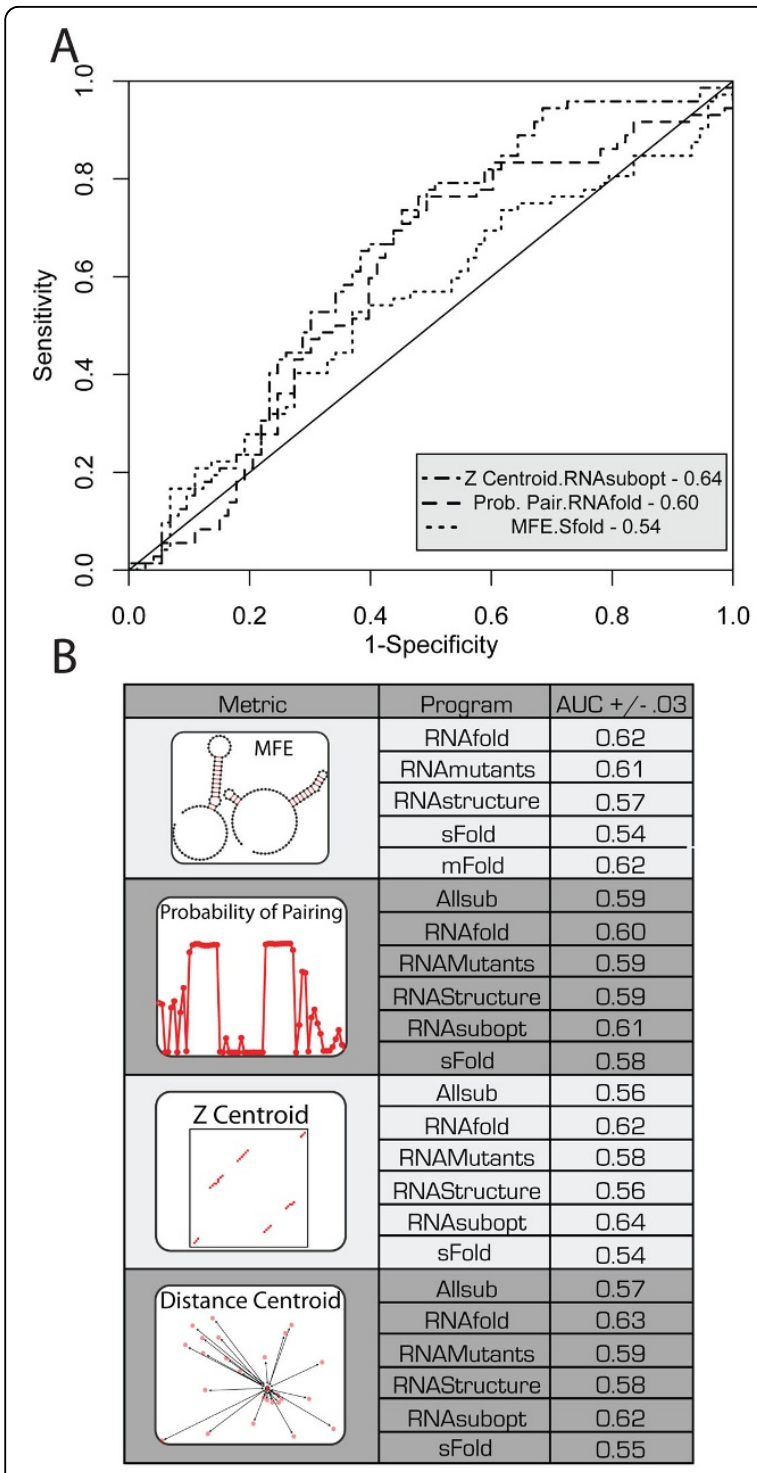

Figure 5 Evaluation of the different pSDC metrics and RNA structure prediction algorithm's performances. A.) Receiver Operator Characteristic (ROC) analysis of three representative metric/algorithm combinations indicating significant differences in the predictive performance. The three curves are for the RNAsubopt prediction of the $Z$ centroid structure (for the first 10,000 structures), base-pair probability as computed from the partition function of RNAfold, and the MFE structures predicted by sFold. B.) Summary of all Area Under the Curve values for ROC analysis of metric/algorithm combinations. In general, most algorithms perform equivalently for identifying the mutations that disrupt RNA structure.

The data we present in Figure 2 are ideal for benchmarking RNA structure prediction algorithms. The analysis we carried out in this manuscript is different from previous secondary structure prediction benchmarks, because we are specifically interested in identifying mutations that globally disrupt a given secondary structure.
We developed metrics based on RNA secondary structure prediction algorithms analogous to our eSDC calculations. We can use such an analogy, since SHAPE data is correlated with base-pair probability. The SDC metrics are purposefully global, and we did not evaluate algorithms for their ability to predict the specific local changes in structure, but rather whether they predict that a specific mutation will disrupt structure relative to others. Our reasoning for this approach is that for the analysis of disease-associated SNPs, we are most interested in identifying the most structurally deleterious mutations.

Although RNA structure prediction algorithms correctly predicted that all RNAs are disrupted by certain mutations, it is clear that predicting exactly which mutation will alter structure remains very challenging. Although there is some variation in the relative performance of the different algorithmic and metric combinations we tested, the AUC values reported in Figure 5B remain relatively low. This result is not necessarily surprising, as none of the RNA structure prediction algorithms (other than RNAmutants) have been optimized to predict which mutations disrupt structure. In fact, an algorithm's sensitivity to point mutations is often viewed as a weakness, favoring methods that are less sensitive to mutation. However, the experimental data clearly show that SNPs can profoundly change an RNA's folding landscape.

The attempts to constantly refine algorithms so as to have them always converge on a single "correct" RNA structure may not improve their ability to identify RiboSNitches. Although only anecdotal, mFold's good performance in our benchmark (AUC 0.62, Figure 5B) may indicate that simpler energy functions, which tend to predict more alternative structures, may ultimately perform better for identifying RiboSNitches. Indeed RNAStructure's relatively low performance in our benchmark is surprising, since it has the most sophisticated and accurate energy function and is most accurate in structure prediction $[48,50]$. Improvements in our ability to predict RiboSNitches will likely require a better understanding of the suboptimal ensemble and how mutations affect it in addition to improved energy functions. With the growing number of sequencing efforts revealing ever more single nucleotide variants in the non-coding regions of the genome, accurate algorithms predicting the structural consequences of these mutations are likely to play an important role in genomic interpretation.

\section{Methods}

\section{Data collection and analysis}

The SHAPE chemical data used in our analysis were downloaded in ISATAB format from the SNRNASM (Single Nucleotide Resolution Nucleic Acid Structure Mapping) and RMDB web sites (http://snrnasm.bio.unc. 
edu and http://rmdb.stanford.edu). The SNRNASM standard was developed to share the results of high-resolution and throughput nucleic acid structure mapping data [58]. We identified RNAs that were probed using SHAPE chemical mapping under standard conditions $(10 \mathrm{mM}$ $\mathrm{MgCl}_{2}$ and $100 \mathrm{mM} \mathrm{NaCl}$ ), and where significant mutational information was available. Only RNAs that were at most two SNPs (or mutations) away from a reference (WT) sequence were considered. The data were normalized as previously described [59], and for the two Riboswitch and P4P6 data sets, manually re-aligned to correct for frameshift errors due to the automated analysis of the data using the HiTRACE software [42]. eSDC values were computed as described by Equation 1:

$$
e S D C=\left(1-{ }^{p} C C\right) \times \sqrt{n}
$$

where ${ }^{p} C C$ is the WT/mutant pearson correlation coefficient and $n$ is the length of the RNA. The eSDC quantitatively evaluates the effect of a mutation on RNA structure. Prior to the calculation of the eSDC, normalized SHAPE values were capped at one in order to increase the metric's ability to reflect changes in structure identified by differences in the peaking pattern and not minor differences in peak intensity. Significance testing for structure disruption was adjusted using a Bonferroni correction.

PCA analysis of the ensemble of structures and clustering Principal components were calculated (as described previously) from a total of 10,000 sampled structures generated equally from a WT sequence and mutants of interest [24]. The principal components were generated from the binary representation of these 10,000 structures. These structures were then projected onto the first two principal components and subjected to the k-means clustering algorithm to reveal distinct clusters [60]. The centroid structure of each cluster was identified from the k-means clustering algorithm and then drawn using R2R [61]. Individual mutant structures were then generated (as discussed in Fig. 3) and projected onto the first two principal components. Each structure projection is colored according to their cluster.

\section{Computation of the partition functions from sampled structures and calculation of the $Z$ centroid}

Partition functions were generated for each ensemble of structures. Each structure is first transformed to matrix form as described in [51]. This is accomplished by creating an $\mathrm{NxN}$ matrix where $\mathrm{N}$ is the length of the sequence and placing a 1 at position $i, j$ and $j, i$ if nucleotides $i$ and $j$ are paired and a 0 if they are not paired. When all the matrices representing the structures are summed together and then divided by the total number of structures, the resulting matrix is the partition matrix. This matrix contains the probability of nucleotide $i$ being paired to $j$. The $\mathrm{Z}$ centroid is defined as the structure with all the probability of pairing for each pair greater than $50 \%$.

\section{ROC analysis of prediction performance}

Each of the program/metric combinations were evaluated using a Receiver Operator Characteristic (ROC) Analysis [62]. The ROC analysis was carried out by calculating the true positive rate (i.e. sensitivity):

$$
T P R=\frac{T P}{T P+F N}
$$

and false positive rate (i.e. 1-specificity):

$$
F P R=\frac{F P}{F P+T N}
$$

from the True Positives (TP), False Positives (FP), True Negatives (TN) and False Negatives (FN):

$$
p S D C=\left(1-{ }^{\text {pred }} C C\right) \times \sqrt{n}
$$

Analogously to the $e S D C$ calculation, we compute a $p S D C$ (predicted Structure Disruption Coefficient) by computing the Pearson Correlation Coefficient $\left({ }^{\text {pred }} \mathrm{CC}\right)$ between WT and mutant for each RNA structure prediction algorithm. This value is analogous to the eSDC in that it allows us to rank order the disruptive effect of mutations on RNA.

To determine ROC values, the mutations were listed from highest to lowest according to their eSDC value. The top $50 \%$ of eSDC values were considered to disrupt the structure while the lowest $50 \%$ preserved structure. A second list was generated using the same mutants but using the pSDC values instead. A true positive was defined as having a pSDC value above a cutoff and experimentally disrupting the structure while a true negative was defined as having a pSDC below a cutoff and experimentally preserving a structure. A false positive or false negative is recorded when the predictions contrast with the experimental results. The pSDC cutoff was defined by stepping through the pSDC ranks. The resulting true positive rates and false positive rates were then used to generate an ROC curve. The area under the curve was calculated for each ROC using the trapezoidal method. This process was bootstrapped for each program/metric 5000 times using 20 randomly selected mutants from each set. Due to the fact that each of the RNA data sets has a differing number of mutants, the bootstrapping is done by sampling 20 mutants from each of the other data sets besides FTL, in order to correct for any bias that might come up due to one program/metric 
favoring one data set over another. This results in the ROC being run on 145 mutants at a time, not the full 470. The average area under the curve was calculated with the standard deviation between runs generating the error. The closer the area under the curve was to one the better the predictive power for a given program/metric.

Precise WT sequences, corresponding mutations (SNPs), eSDC values and normalized SHAPE data are provided as separate excel spreadsheets in the additional files. These data should facilitate further benchmarking efforts for novel algorithms to predict RNA structure change.

\section{Additional material}

Additional file 1: eSDC and SHAPE data for the FTL 199 nucleotide length RNA construct.

Additional file 2: eSDC and SHAPE data for the FTL 226 nucleotide length RNA construct.

Additional file 3: eSDC and SHAPE data for the Adenine Riboswitch RNA construct.

Additional file 4: eSDC and SHAPE data for Glycine Riboswitch without Glycine RNA construct.

Additional file 5: eSDC and SHAPE data for the Glycine Riboswitch with Glycine RNA construct.

Additional file 6: eSDC and SHAPE data for the GMP Riboswitch with CDM RNA construct.

Additional file 7: eSDC and SHAPE data for the GMP Riboswitch without CDM RNA construct.

Additional file 8: eSDC and SHAPE data for the P4P6 subdomain of the Tetrahymena thermophila group I intron RNA construct.

\section{List of abbreviations}

eSDC: experimental Structure Disruption Coefficientl; PSDC: predicted Structure Disruption Coefficient; SHAPE: Selective 2'-Hydroxyl Acylation analyzed by Primer Extension; SNP: Single Nucleotide Polymorphism; FPR: False Positive Rate; TPR: True Positive Rate; SNRNASM: Single Nucleotide Resolution Nucleic Acid Structure Mapping

\section{Acknowledgments}

We would like to thank Rhiju Das and Pablo Cordero (Stanford University) for providing SHAPE data sets in the SNRNASM format through their database (http://rmdb.stanford.edu/). This work is supported by grants R0O GM079953 (NIGMS), R21 MH087336 (NIMH), R01 HL111527 and R01 GM101237 to A.L.

This article has been published as part of BMC Genomics Volume 13 Supplement 4, 2012: SNP-SIG 2011: Identification and annotation of SNPs in the context of structure, function and disease. The full contents of the supplement are available online at http://www.biomedcentral.com/ bmcgenomics/supplements/13/S4.

\section{Authors' contributions}

JR performed the analysis of the SHAPE data, determined statistical significance and conceived the statistical tests. JM performed the structural predictions, made Figures 3 and 4 . AL directed the research and wrote the manuscript.

\section{Competing interests}

The authors declare that they have no competing interests.
References

1. Preall JB, Sontheimer EJ: RNAi: RISC gets loaded. Cell 2005, 123(4):543-545.

2. Pesole G, Liuni S, Grillo G, Ippedico M, Larizza A, Makalowski W, Saccone C: UTRdb: a specialized database of $5^{\prime}$ and $3^{\prime}$ untranslated regions of eukaryotic mRNAs. Nucleic Acids Res 1999, 27(1):188-191.

3. Laederach A, Chan JM, Schwartzman A, Willgohs E, Altman RB: Coplanar and coaxial orientations of RNA bases and helices. Rna 2007, 13(5):643-650.

4. Laederach $\mathrm{A}$ : Informatics challenges in structured RNA. Briefings in bioinformatics 2007, 8(5):294-303.

5. Doherty EA, Doudna JA: Ribozyme structures and mechanisms. Annu Rev Biophys Biomol Struct 2001, 30:457-475.

6. Martin JS, Simmons K, Laederach A: Exhaustive Enumeration of Kinetic Model Topologies for the Analysis of Time-Resoved RNA Folding. Algorithms 2009, 2(1):200-214.

7. Laederach A, Shcherbakova I, Liang MP, Brenowitz M, Altman RB: Local kinetic measures of macromolecular structure reveal partitioning among multiple parallel pathways from the earliest steps in the folding of a large RNA molecule. J Mol Biol 2006, 358(4):1179-1190.

8. Laederach A, Shcherbakova I, Jonikas MA, Altman RB, Brenowitz M: Distinct contribution of electrostatics, initial conformational ensemble, and macromolecular stability in RNA folding. Proc Natl Acad Sci U S A 2007, 104(17):7045-7050.

9. Das R, Kwok LW, Millett IS, Bai Y, Mills TT, Jacob J, Maskel GS, Seifert S, Mochrie SG, Thiyagarajan $P$, et al: The fastest global events in RNA folding: electrostatic relaxation and tertiary collapse of the Tetrahymena ribozyme. J Mol Biol 2003, 332(2):311-319.

10. Chu VB, Herschlag D: Unwinding RNA's secrets: advances in the biology, physics, and modeling of complex RNAs. Curr Opin Struct Biol 2008, 18(3):305-314

11. Zwarts KY, Clee SM, Zwinderman AH, Engert JC, Singaraja R, Loubser O, James E, Roomp K, Hudson TJ, Jukema JW, et al: ABCA1 regulatory variants influence coronary artery disease independent of effects on plasma lipid levels. Clin Genet 2002, 61(2):115-125.

12. Kimchi-Sarfaty C, Oh JM, Kim IW, Sauna ZE, Calcagno AM, Ambudkar SV Gottesman MM: A "silent" polymorphism in the MDR1 gene changes substrate specificity. Science 2007, 315(5811):525-528.

13. Showalter HD, Denny WA: A roadmap for drug discovery and its translation to small molecule agents in clinical development for tuberculosis treatment. Tuberculosis (Edinburgh, Scotland) 2008, 88(Suppl 1):S3-17.

14. Kohnlein T, Welte T: Alpha-1 antitrypsin deficiency: pathogenesis, clinical presentation, diagnosis, and treatment. The American journal of medicine 2008, 121(1):3-9.

15. Glinsky GV: SNP-guided microRNA maps (MirMaps) of 16 common human disorders identify a clinically accessible therapy reversing transcriptional aberrations of nuclear import and inflammasome pathways. Cell cycle (Georgetown, Tex 2008, 7(22):3564-3576.

16. Weinstock GM: ENCODE: more genomic empowerment. Genome Res 2007 17(6):667-668.

17. Karolchik D, Kuhn RM, Baertsch R, Barber GP, Clawson H, Diekhans M, Giardine B, Harte RA, Hinrichs AS, Hsu F, et al: The UCSC Genome Browser Database: 2008 update. Nucleic Acids Res 2008, 36(Database issue): D773-779.

18. Reeder J, Hochsmann M, Rehmsmeier M, Voss B, Giegerich R: Beyond Mfold: recent advances in RNA bioinformatics. J Biotechnol 2006, 124(1):41-55.

19. Kertesz M, Wan Y, Mazor E, Rinn JL, Nutter RC, Chang HY, Segal E: Genomewide measurement of RNA secondary structure in yeast. Nature 2010, 467(7311):103-107

20. Ying SY, Chang DC, Miller JD, Lin SL: The microRNA: overview of the RNA gene that modulates gene functions. Methods Mol Biol 2006, 342:1-18.

21. Tucker BJ, Breaker RR: Riboswitches as versatile gene control elements. Curr Opin Struct Biol 2005, 15(3):342-348.

22. Lemay JF, Lemieux C, St-Andre O, Bachand F: Crossing the borders: poly (A)-binding proteins working on both sides of the fence. RNA Biol 2010, 7(3):291-295.

23. Ding $Y$, Chan CY, Lawrence CE: Sfold web server for statistical folding and rational design of nucleic acids. Nucleic Acids Res 2004, 32(Web Server issue):W135-141 
24. Halvorsen M, Martin JS, Broadaway S, Laederach A: Disease-associated mutations that alter the RNA structural ensemble. PLoS genetics 2010, 6(8):e1001074.

25. Gorlov IP, Gorlova OY, Sunyaev SR, Spitz MR, Amos Cl: Shifting paradigm of association studies: value of rare single-nucleotide polymorphisms. American journal of human genetics 2008, 82(1):100-112.

26. $\mathrm{Ng} \mathrm{PC}$, Henikoff S: SIFT: Predicting amino acid changes that affect protein function. Nucleic Acids Res 2003, 31(13):3812-3814.

27. $\mathrm{Ng} P$ PC, Henikoff S: Predicting deleterious amino acid substitutions. Genome Res 2001, 11(5):863-874.

28. Churkin A, Gabdank I, Barash D: The RNAmute web server for the mutational analysis of RNA secondary structures. Nucleic Acids Res 2011, 39(Web Server issue):W92-99.

29. Barash D, Churkin A: Mutational analysis in RNAs: comparing programs for RNA deleterious mutation prediction. Briefings in bioinformatics 2011, 12(2):104-114.

30. Glinsky GV: Disease phenocode analysis identifies SNP-guided microRNA maps (MirMaps) associated with human "master" disease genes. Cell cycle (Georgetown, Tex 2008, 7(23):3680-3694.

31. Das R, Kudaravalli M, Jonikas M, Laederach A, Fong R, Schwans JP, Baker D, Piccirilli JA, Altman RB, Herschlag D: Structural inference of native and partially folded RNA by high-throughput contact mapping. Proc Natl Acad Sci U S A 2008, 105(11):4144-4149.

32. Das R, Karanicolas J, Baker D: Atomic accuracy in predicting and designing noncanonical RNA structure. Nat Methods 2010, 7(4):291-294.

33. Weinberg Z, Barrick JE, Yao Z, Roth A, Kim JN, Gore J, Wang JX, Lee ER, Block KF, Sudarsan N, et al: Identification of 22 candidate structured RNAs in bacteria using the CMfinder comparative genomics pipeline. Nucleic Acids Res 2007, 35(14):4809-4819.

34. Lipfert J, Das R, Chu VB, Kudaravalli M, Boyd N, Herschlag D, Doniach S: Structural transitions and thermodynamics of a glycine-dependent riboswitch from Vibrio cholerae. J Mol Biol 2007, 365(5):1393-1406.

35. Lemay JF, Penedo JC, Mulhbacher J, Lafontaine DA: Molecular basis of RNA-mediated gene regulation on the adenine riboswitch by singlemolecule approaches. Methods Mol Biol 2009, 540:65-76.

36. Lemay JF, Lafontaine DA: Core requirements of the adenine riboswitch aptamer for ligand binding. Rna 2007, 13(3):339-350.

37. Lemay JF, Penedo JC, Tremblay R, Lilley DM, Lafontaine DA: Folding of the adenine riboswitch. Chem Biol 2006, 13(8):857-868.

38. Rocca-Serra P, Bellaousov S, Birmingham A, Chen C, Cordero P, Das R, Davis-Neulander L, Duncan CD, Halvorsen M, Knight R, et al: Sharing and archiving nucleic acid structure mapping data. RNA 2011, 17(7):1204-1212.

39. Wilkinson KA, Merino EJ, Weeks KM: Selective 2'-hydroxyl acylation analyzed by primer extension (SHAPE): quantitative RNA structure analysis at single nucleotide resolution. Nature protocols 2006, 1(3):1610-1616.

40. Wilkinson KA, Vasa SM, Deigan KE, Mortimer SA, Giddings MC, Weeks KM: Influence of nucleotide identity on ribose 2'-hydroxyl reactivity in RNA. Rna 2009.

41. Jones CN, Wilkinson KA, Hung KT, Weeks KM, Spremulli LL: Lack of secondary structure characterizes the $5^{\prime}$ ends of mammalian mitochondrial mRNAs. RNA 2008, 14(5):862-871.

42. Yoon S, Kim J, Hum J, Kim H, Park S, Kladwang W, Das R: HiTRACE: highthroughput robust analysis for capillary electrophoresis. Bioinformatics 2011, 27(13):1798-1805

43. Ferrari F, Foglieni B, Arosio P, Camaschella C, Daraio F, Levi S, Garcia Erce JA, Beaumont C, Cazzola M, Ferrari M, et al: Microelectronic DNA chip for hereditary hyperferritinemia cataract syndrome, a model for largescale analysis of disorders of iron metabolism. Human mutation 2006, 27(2):201-208

44. Cremonesi L, Paroni R, Foglieni B, Galbiati S, Fermo I, Soriani N, Belloli S, Ruggeri G, Biasiotto G, Cazzola M, et al: Scanning mutations of the 5'UTR regulatory sequence of $\mathrm{L}$-ferritin by denaturing high-performance liquid chromatography: identification of new mutations. British journal of haematology 2003, 121(1):173-179.

45. Lemay JF, Desnoyers G, Blouin S, Heppell B, Bastet L, St-Pierre P, Masse E, Lafontaine DA: Comparative study between transcriptionally- and translationally-acting adenine riboswitches reveals key differences in riboswitch regulatory mechanisms. PLoS genetics 2011, 7(1):e1001278.
46. Grundy FJ, Henkin TM: From ribosome to riboswitch: control of gene expression in bacteria by RNA structural rearrangements. Crit Rev Biochem Mol Biol 2006, 41(6):329-338.

47. Waldispuhl J, Clote P: Computing the partition function and sampling for saturated secondary structures of RNA, with respect to the Turner energy model. J Comput Biol 2007, 14(2):190-215.

48. Mathews DH: Using an RNA secondary structure partition function to determine confidence in base pairs predicted by free energy minimization. RNA 2004, 10(8):1178-1190.

49. Hofacker IL, Stadler PF: Memory efficient folding algorithms for circular RNA secondary structures. Bioinformatics 2006, 22(10):1172-1176.

50. Wilkinson KA, Gorelick RJ, Vasa SM, Guex N, Rein A, Mathews DH, Giddings MC, Weeks KM: High-throughput SHAPE analysis reveals structures in HIV-1 genomic RNA strongly conserved across distinct biological states. PLOS biology 2008, 6(4):e96.

51. Ding Y, Chan CY, Lawrence CE: RNA secondary structure prediction by centroids in a Boltzmann weighted ensemble. Rna 2005, 11(8):1157-1166.

52. Cloonan N, Grimmond SM: Transcriptome content and dynamics at single-nucleotide resolution. Genome biology 2008, 9(9):234.

53. Ashraf SI, Kunes S: A trace of silence: memory and microRNA at the synapse. Curr Opin Neurobiol 2006, 16(5):535-539.

54. Lu C, Smith AM, Fuchs RT, Ding F, Rajashankar K, Henkin TM, Ke A: Crystal structures of the SAM-III/S(MK) riboswitch reveal the SAM-dependent translation inhibition mechanism. Nat Struct Mol Biol 2008, 15(10):1076-1083.

55. Gilbert SD, Rambo RP, Van Tyne D, Batey RT: Structure of the SAM-II riboswitch bound to S-adenosylmethionine. Nat Struct Mol Biol 2008, 15(2):177-182.

56. Laederach A, Shcherbakova I, Liang M, Brenowitz M, Altman RB: Local kinetic measures of macromolecular structure reveal partitioning among multiple parallel pathways from the earliest steps in the folding of a large RNA molecule. J Mol Biol 2006, 358(358):1179-1190.

57. Sadee W, Wang D, Papp AC, Pinsonneault JK, Smith RM, Moyer RA, Johnson AD: Pharmacogenomics of the RNA world: structural RNA polymorphisms in drug therapy. Clin Pharmacol Ther 2011, 89(3):355-365.

58. Rocca-Serra P, Bellaousov S, Birmingham A, Chen C, Cordero P, Das R, Davis-Neulander L, Duncan CD, Halvorsen M, Knight R, et al: Sharing and archiving nucleic acid structure mapping data. RNA 2011.

59. Quarrier S, Martin JS, Davis-Neulander L, Beauregard A, Laederach A: Evaluation of the information content of RNA structure mapping data for secondary structure prediction. RNA 2010, 16(6):1108-1117.

60. Tibshirani RJ, Walther G, Hastie T: Estimating the number of clusters in a data set via the gap statistic. Journal of the Royal Society: Series $B$ (Statistical Methodology) 2001, 63(2):411-423.

61. Weinberg Z, Breaker RR: R2R-software to speed the depiction of aesthetic consensus RNA secondary structures. BMC bioinformatics 2011, 12:3.

62. Sonego $\mathrm{P}$, Kocsor A, Pongor S: ROC analysis: applications to the classification of biological sequences and 3D structures. Briefings in bioinformatics 2008, 9(3):198-209.

63. Mitra S, Laederach A, Golden BL, Altman RB, Brenowitz M: RNA molecules with conserved catalytic cores but variable peripheries fold along unique energetically optimized pathways. RNA 2011, 17(8):1589-1603.

64. Tafer H, Ameres SL, Obernosterer G, Gebeshuber CA, Schroeder R, Martinez J, Hofacker IL: The impact of target site accessibility on the design of effective siRNAs. Nature biotechnology 2008, 26(5):578-583.

doi:10.1186/1471-2164-13-S4-S6

Cite this article as: Ritz et al:: Evaluating our ability to predict the structural disruption of RNA by SNPs. BMC Genomics 2012 13(Suppl 4):S6. 